\title{
Improving SFR Economics Through Innovations from Thermal Design and Analysis Aspects
}

\section{ICAPP 2008}

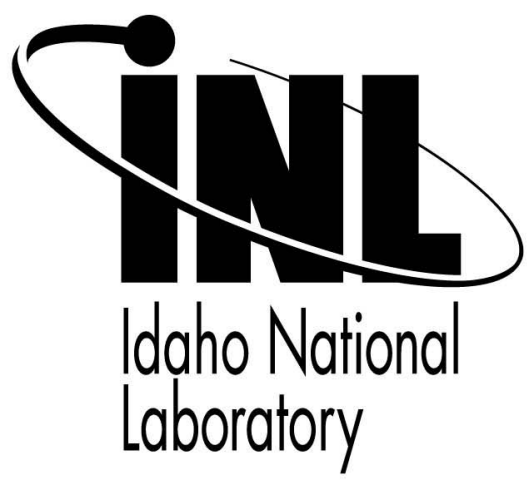

Haihua Zhao

Hongbin Zhang

Vincent A. Mousseau

Per F. Peterson

\author{
June 2008
}

This is a preprint of a paper intended for publication in a journal or proceedings. Since changes may be made before publication, this preprint should not be cited or reproduced without permission of the author. This document was prepared as an account of work sponsored by an agency of the United States Government. Neither the United States Government nor any agency thereof, or any of their employees, makes any warranty, expressed or implied, or assumes any legal liability or responsibility for any third party's use, or the results of such use, of any information, apparatus, product or process disclosed in this report, or represents that its use by such third party would not infringe privately owned rights. The views expressed in this paper are not necessarily those of the United States Government or the sponsoring agency. 


\title{
Improving SFR Economics through Innovations from Thermal Design and Analysis Aspects
}

\author{
Haihua Zhao ${ }^{\mathrm{a} *}$, Hongbin Zhang ${ }^{\mathrm{a}}$, Vincent A. Mousseau ${ }^{\mathrm{a}}$, and Per F. Peterson ${ }^{\mathrm{b}}$ \\ ${ }^{a}$ Idaho National Laboratory, USA \\ ${ }^{b}$ Department of Nuclear Engineering, University of California, Berkeley, USA \\ * Corresponding author: Idaho National Laboratory, PO BOX 1625, Idaho Falls, ID 83415-3870, USA \\ Tel: +1 (208) 526-2679, Fax: +1 (208) 526-0528, Email: Haihua.Zhao@inl.gov
}

\begin{abstract}
Achieving economic competitiveness as compared to LWRs and other Generation IV (Gen-IV) reactors is one of the major requirements for large-scale investment in commercial sodium cooled fast reactor (SFR) power plants. Advances in R\&D for advanced SFR fuel and structural materials provide key long-term opportunities to improve SFR economics. In addition, other new opportunities are emerging to further improve SFR economics. This paper provides an overview on potential ideas from the perspective of thermal hydraulics to improve SFR economics. These include a new hybrid loop-pool reactor design to further optimize economics, safety, and reliability of SFRs with more flexibility, a multiple reheat and intercooling helium Brayton cycle to improve plant thermal efficiency and reduce safety related overnight and operation costs, and modern multi-physics thermal analysis methods to reduce analysis uncertainties and associated requirements for over-conservatism in reactor design. This paper reviews advances in all three of these areas and their potential beneficial impacts on SFR economics.
\end{abstract}

\section{INTRODUCTION}

The sodium cooled fast reactor (SFR) has been studied since the early period of nuclear energy development. Recently SFRs have been proposed to be used as actinide burners to close the nuclear fuel cycle ${ }^{1}$. Achieving economic competitiveness as compared to LWRs and other Generation IV (Gen-IV) reactors is one of major requirements for large-scale investment in commercial SFR power plants. However, even advanced SFR designs are anticipated to be more expensive than the current light water reactors. This perception contradicts the economic analysis results for some advanced SFR designs, such as European Fast Reactor (EFR) ${ }^{2}$, and US Advanced Liquid Metal Reactor (ALMR) ${ }^{3}$ basing on General Electric Company's PRISM ${ }^{4}$ design. Both programs predicted that advanced SFRs could achieve similar generating cost as LWRs. However, when reading those papers carefully, one can find that to draw the above conclusion, both economic analyses assumed that the SFR and LWR must have similar capacity factors and the power levels.

While very large SFR plants with similar power level as current large LWR plants can be constructed, like the Superphénix plant ${ }^{5}$, achieving similar high capacity factors as current LWRs (about $90 \%$ in the US) has never been demonstrated for any SFR plants. Although successful operating experience with past and existing test and prototype SFRs has demonstrated generally good safety and reliability, incidents and failures did occur in these prototype reactors and plants ${ }^{5}$. The Russian BN-600 achieved $75 \%$ capacity factor, which is the best record for SFRs. Even it had recorded a number of sodium leakage incidents, some involved sodium-water reactions. These incidents limited the SFR's capability to achieve high availability. Therefore, to achieve competitive SFR economics, the reliability and availability of SFRs must be further improved. Additional cost reductions can be achieved by reduced plant overnight cost (the cost of a construction project if no interest was incurred during construction) and operational cost, including fuel cycle cost.

Current LWR fleets in the US and several other developed countries have consistently achieved a capacity factor above $90 \%$. This can be largely attributed to effective in-service inspection (ISI) and maintenance. Some potential accidents have been prevented by refueling outage inspections. SFR plants need to be designed to facilitate ISI and maintenance so that high availability can be achieved. Design features are also needed to enhance high reliability, such as guard pipes and vessels to provide 
double barriers to prevent sodium leakage, double-walled steam generators to prevent sodium-water reactions, or the replacement of steam cycles with advanced Brayton cycles to eliminate steam generators. Although an overnight cost lower than LWRs could be achieved for some advanced SFR designs, such as EFR, it is still possible to further reduce SFR plant overnight cost through innovative designs. Operational cost can also be reduced by innovations. Reducing the overnight cost and enhancing safety and reliability must be considered at the same time. In the past decade, Japanese researchers made impressive effort to improve their advanced loop type SFR design through JFSR project ${ }^{6,7}$, more or less with evolutionary methods. Todreas ${ }^{8}$ summarized thermal hydraulic challenges in fast reactor design. However, few potential cost reduction solutions were provided.

Advances in R\&D for advanced SFR fuel ${ }^{9}$ and structural materials ${ }^{7}$ provide key long-term opportunities to improve SFR economics. In addition, other new opportunities are emerging to further improve SFR economics. This paper provides an overview on potential ideas from the perspective of thermal hydraulics to improve SFR economics. All those ideas were originated outside of the traditional SFR field. These include a new hybrid loop-pool reactor design to further optimize economics, safety, and reliability of SFRs with increased flexibility $^{10}$, a multiple reheat and intercooling helium Brayton cycle to improve plant thermal efficiency and reduce safety related overnight and operation $\operatorname{costs}^{11}$, and modern multi-physics thermal analysis methods to reduce analysis uncertainties and associated requirements for over-conservatism in reactor design ${ }^{12}$.

By borrowing the loop-in-a-tank concept originally proposed as a design variant of the liquid salt cooled Advanced High Temperature Reactor (AHTR) ${ }^{13}$, a hybrid loop-pool SFR design was proposed ${ }^{10}$, with the goal of reducing capital cost and improving safety. The design takes advantage of the easier in-service inspection and compactness of loop designs and the inherent safety of pool designs. Primary loops are formed by connecting hot sodium at the reactor outlet plenum (hot pool), intermediate heat exchangers (IHX), primary pumps and reactor inlet plenum with pipes. The primary loops are immersed in the cold pool (buffer pool), which provides an extra safety barrier and large thermal inertia. During accidents, the modular Pool Reactor Auxiliary Cooling System (PRACS) transfers heat from the reactor core to the cold pool. This hybrid loop-pool SFR design could potentially increase the core inlet and outlet temperatures. This would result in higher thermal efficiencies without having a negative impact on the passive safety features. In addition the hybrid loop-pool design enables the use of compact heat exchangers as the IHX, potentially reducing the size of the primary system. The hybrid design could also facilitate in-service inspection so that reliability can be increased.

Another opportunity to improve the economics of the SFR is to replace the traditional steam Rankine cycle and recently proposed supercritical $\mathrm{CO}_{2}$ Brayton cycle (SCO2) with a helium multiple reheat Brayton cycle ${ }^{11}$. Recent studies show similar or better thermal efficiency than steam and $\mathrm{SCO} 2$ cycles. The major advantage comes from the use of inert helium (or nitrogen) as the power conversion working fluid, which eliminates the requirement for safety systems to prevent and mitigate the sodium-water and sodium- $\mathrm{CO}_{2}$ reactions.

The economics can also be improved by reducing safety analysis uncertainty and the associated requirements for over-conservatism in design through high fidelity multi-scale, multi-physics thermal hydraulic analysis methods. Advances in numerical methods during the past two decades make it possible to perform simulations with second order accuracy in both time and space. New methods also enable implicitly coupled solution algorithms between coolant thermal hydraulics, solid conduction, and neutronics, which can greatly reduce numerical errors in simulations for very fast or very long transients. Other progresses such as higher dimensional methods than conventional system codes used could also reduce uncertainties, such as CFD simulations for components with strong 3-D effects and 1-D transient models for pool thermal stratification.

This paper reviews advances in all three of these areas and their potential beneficial impacts on SFR economics.

\section{AN INNOVATIVE HYBRID LOOP-POOL SFR DESIGN}

\section{II.A. Pool Design versus Loop Design}

The existing SFRs have two types of designs - loop type and pool type. In the loop type designs, such as $\mathrm{JOYO}^{14}$, MONJU ${ }^{15}$, and $\mathrm{JSFR}^{6}$ in Japan, the primary coolant is circulated through IHXs and pumps external to the reactor tank. The major advantages of the loop design include compactness and easier in-service inspection and maintenance. The disadvantage is higher possibility of sodium leakage than a pool design. ${ }^{16}$

In the pool type designs such as EBR-II and ALMR (USA), BN-600M (Russia), Superphénix (France) and EFR $(E U)^{5}$, the reactor core, primary pumps, IHXs and direct reactor auxiliary cooling system (DRACS) heat exchangers (DHX) all are immersed in a pool of sodium coolant within the reactor vessel, making a loss of primary 
coolant accident extremely unlikely. However, a pool type design requires a larger reactor vessel and has more complex internal structures. Table I compares loop and pool SFR designs, mainly based on the opinions from references 1, 5, and 16. The comparison only reflects general trends in typical loop and pool SFR designs. Fundamental technologies are common for both loop type and pool type. ${ }^{16}$

\section{TABLE I}

Comparison of Typical Pool and Loop SFR designs

\begin{tabular}{|l|l|l|}
\hline & \multicolumn{1}{|c|}{ Pool Design } & \multicolumn{1}{c|}{ Loop Design } \\
\hline Economics & • Simple large vessel & - Smaller vessels but \\
& - Smaller reactor & with more \\
& building & connection pipes \\
& - In vessel spent fuel & - Larger reactor \\
& storage & building \\
Safety & - Slower and milder & - Faster transients due \\
& transients & to less thermal \\
& - Reduced possibility of & inertia \\
& core uncovery & - Higher possibility of \\
& & Na leakage \\
Operation & - More difficult to & - Easier for ISI and \\
and & perform ISI & repair \\
Inspection & - Allow leaky loops and & - Require shielding \\
& flexible connections & for external loops \\
\hline
\end{tabular}

In a typical pool SFR design, the hot sodium at core outlet temperature in the hot pool is separated from the cold sodium at the core inlet temperature in the cold pool by a single integrated structure called a redan. The redan provides the exchange of the hot sodium from the hot pool to the cold pool through the IHXs. During loss of forced circulation (LOFC) transients, small flow resistance is essential to establish adequate natural circulation to remove heat from the reactor to the cold pool. Due to this reason, only traditional tube and shell IHXs with low flow resistance can be used. To increase the thermal cycle efficiency of advanced Brayton cycles, both the reactor inlet and outlet temperatures need to be further increased. Because the reactor inlet temperature is approximately the cold pool temperature, increasing the cold pool temperature would: (1) decrease thermal inertia of the system, and (2) the reactor vessel would be subjected to higher temperatures in either normal and transient conditions. To meet safety requirements, the reactor inlet temperature may have to be set at a lower value, sacrificing power conversion efficiency.

\section{II.B. Hybrid Loop-Pool SFR Design}

The new hybrid loop-pool design ${ }^{10}$ takes advantage of the compactness and easier in-service inspection of the loop design ${ }^{16}$ and the inherent safety of the pool design ${ }^{1}$. A closed primary loop is created by connecting hot sodium at the reactor outlet plenum (hot pool), IHXs, primary pumps and reactor inlet plenum with pipes. The primary loops are immersed in the cold pool (buffer pool). During accidents, the modular PRACS transfers heat from the reactor core to the cold pool.

Fig. 1 compares conventional pool design and the new hybrid loop-pool design configurations. Under normal operation, the primary loops operate in forced circulation driven by primary pumps which could be located either in the reactor hot leg or in the cold leg. The IHXs could be either traditional tube-shell heat exchangers or compact heat exchangers. Compact heat exchangers have a much higher power density (5 to 10 times higher) and are much smaller than tube-shell type heat exchangers.
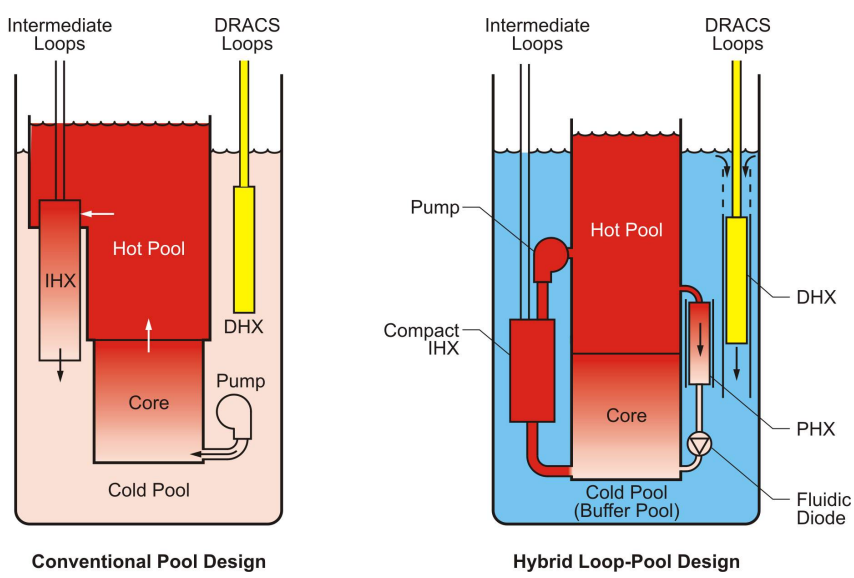

Fig. 1. Comparison of conventional pool design and innovative hybrid loop-pool design for SFR.

The primary system and the cold pool are thermally coupled by the PRACS, which is composed of PRACS heat exchangers (PHX), fluidic diodes and connecting pipes. A fluidic diode reduces leakage flows under primary loop forced circulation. Fluidic diodes are simple, passive devices that provide large flow resistance in one direction and small flow resistance in the reverse direction. The simplest fluidic diode devices generate an irreversible loss of kinetic energy by creating a strong vortex flow in one direction, while flow in the opposite direction does not have this effect. Fluid diode devices have also been proposed to be used in Japanese advanced loop SFR designs, ${ }^{6,7}$ where fluid diodes in the DRACS loops reduce the bypass flow under normal operation. PHX modules use conventional tube bundles to reduce flow resistance and are in baffles to enhance natural circulation as shown in Fig. 1.

For normal power operation with forced cooling, the primary loops transfer heat to modular IHXs located in the cold pool. A small bypass with reactor inlet temperature 
flows upward through PHXs. This bypass flow transfers heat to the cold pool, as well as additional heat transfer through the primary loop pipe and component walls. This added heat is mainly removed by the DRACS to the environment so that the cold pool temperature remains constant. Under a loss of heat sink transient where the primary pumps continue to operate, this bypass flow provides the capability to remove decay heat to the cold pool.

Under the LOFC transient with reactor scram, reduced heat transfer in the reactor core causes the core temperatures to rise. Natural circulation establishes quickly and flow reversal occurs in the PRACS loops. Due to higher flow resistance in IHXs and stopped pumps and due to loss of secondary heat sink, natural circulation through IHXs is not important for decay heat removal compared to that by the PRACS. Decay heat removal mainly occurs through the PHX modules. The PHX heat transfer area is sized to match decay heat generation approximately 2 to 3 hours after LOFC occurs. The DRACS heat removal systems are sized to match decay heat generation approximately 4 to 6 hours after LOFC occurs.

An additional passive safety system could also be used to enhance passive safety and provide backup, for example, the Indirect Reactor Auxiliary Cooling System (IRACS) used in the JSFR design ${ }^{5,6}$. IRACS removes the decay heat by natural circulations through the primary loops and part of the intermediate heat transfer loops. Due to the large flow area in a compact IHX, the natural circulations even through a compact IHX can still provide enough flow for the decay heat removal in the longer term when the required flow rate is small relative to the flow through PRACS. Future study will provide an IRACS design and quantitative analysis for the system.

\section{II.C. Advantages of the Hybrid Loop-Pool SFR Design}

This new hybrid loop-pool SFR design has the potential to improve SFR economics and safety from the following aspects:

- Flexibility to optimize system design - Primary loops and the passive safety system PRACS are fully decoupled, so are the reactor inlet and the cold pool temperatures. Decoupling the primary loops and the passive safety loops were suggested by Koch according to his EBR-II experience. ${ }^{17}$ The cold pool and the hot pool are physically separated. The cold pool becomes a buffer pool. More freedom is available to optimize the design by improving economics while meeting safety requirements. For example, the natural circulation provided by the PRACS can be stronger than the natural circulation only through the primary loop in a conventional pool design due to the reduced flow resistance and increased buoyancy force. The increased natural circulation flow would reduce the peak clad temperature during LOFC transients. Effective cooling of the fuel rods is not only determined by the reactor inlet temperature, but equally importantly by the flow rate. If the flow rate becomes zero in the core, the sodium will begin to boil within a very short time irrespective of the reactor inlet temperature.

- Cost reduction - Both plant equipment cost and operational cost can be potentially reduced with this new design. For example, this design allows more compact IHXs to be used which offer several potential benefits: (1) smaller logarithmic mean temperature difference (LMTD) across the more compact IHX can provide higher heating temperature for the power conversion system so that the thermal efficiency is increased; (2) the smaller IHX size and less mass could imply lower IHX cost; (3) the buffer pool tank size and related building size could be reduced for large power reactor designs. The primary sodium inventory is significantly reduced by decoupling the buffer pool from the primary cooling circuit. Consequently, less radioactive sodium has to be purified during operation. Both core inlet and outlet temperatures could be increased without affecting safety, which yields a higher thermal efficiency for electricity generation.

- Facilitating in-service inspection and maintenance This design offers a new approach for the ISI. In the conventional pool type SFR design, all the primary loop components are immersed in primary sodium. Sodium is an opaque fluid and in-sodium inspection is difficult. Due to the fact that all fuel assemblies need to be removed before the primary system can be drained, inspection after draining the primary system will only happen a few times in the whole plant life. For the new design, one major ISI can be performed in this way: decay heat can be removed by running primary and intermediate loops at low flow rates for several days before the decay heat drops to very low level; the buffer sodium is pumped out and all the primary boundary and buffer tank inner surface now are exposed; a remote operated vehicle (ROV) is then sent in to perform inspection as shown in Fig. 2. The environment for the ROV is mild: low temperature and low-radioactivity. To meet the safety regulatory requirements, alternative passive safety such as IRACS and detailed procedures and risk analyses are needed before adopting such an ISI approach.

- Safety enhancement - Enhanced natural circulation through the decoupled safety system could reduce the 
peak clad temperature and maximum sodium temperature during transients as discussed before. The cold pool temperature can be set at a lower value than a conventional pool design, which increases thermal inertia. Having the closed primary loops immersed in a secondary tank design provides an extra barrier to prevent primary sodium leakage and reduces the probability of core uncovery.

- Scalability: The modularity of the IHXs, PRACS, and DRACS allows the plant design to be easily scaled up to larger power levels to take advantage of the economics of scale. The experience and data from a small pilot plant can be used to license a large commercial plant with very small scaling distortion.

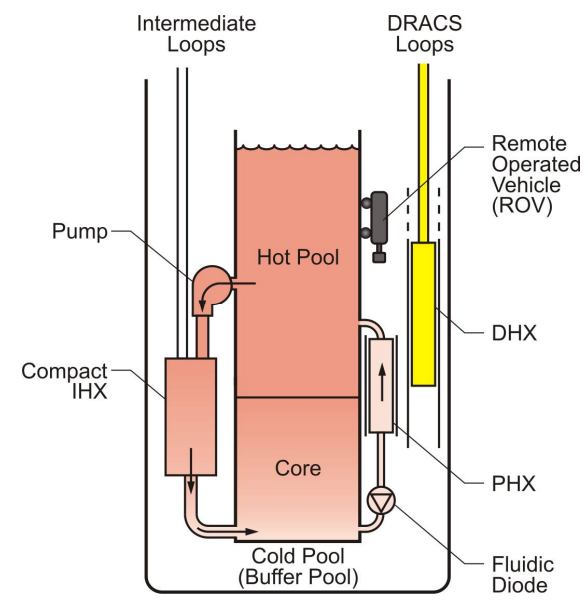

Fig. 2. Schematics of inspecting all the exposed primary boundary and buffer tank by ROV after buffer tank fluid is drained.

The above discussions only summarize some potential benefits for the new hybrid design. These benefits need further detailed study to confirm and future trade-off investigations will give more insights on the implications of the new hybrid design to the SFR economics, reliability and safety.

\section{II.D. LOFC Analysis Results}

In order to verify that the PRACS can effectively transfer heat from the primary loop to the buffer pool, LOFC transient analyses were performed with a lumped volume based method and RELAP5-3 $\mathrm{D}^{18}$. In the analyses, the Advanced Burner Test Reactor (ABTR) developed by Argonne National Laboratory ${ }^{1}$ was used as the reference reactor design. ABTR is a pool type design. With some straightforward modifications to the ABTR design, such as adding the PRACS and closing the primary loops as shown in Fig. 1, a hybrid loop-pool design, SFR-Hybrid, is obtained. The SFR-Hybrid core, IHX, and primary pump designs are the same as that for ABTR. Reactor inlet/outlet temperatures are $355^{\circ} \mathrm{C} / 510^{\circ} \mathrm{C}$.

Simplified calculations which treat the primary loops and the cold pool as two interconnected lumped masses provide a first-order estimate for the transient response that follows LOFC. Fig. 3 shows the resulting temperature and power histories, for the case where the PHX modules are sized with a nominal heat removal ability at $1.3 \%$ of normal reactor power, and the DHX with a capacity of $0.7 \%$ of normal reactor power. The buffer pool average temperature is set at $300^{\circ} \mathrm{C}$. The effects of the large thermal capacity provided by hot pool in the primary loop and by the cold pool are readily seen, with the volume averaged temperature of the primary loop rising less than $50^{\circ} \mathrm{C}$ above the initial value of $488^{\circ} \mathrm{C}$ during the transient.

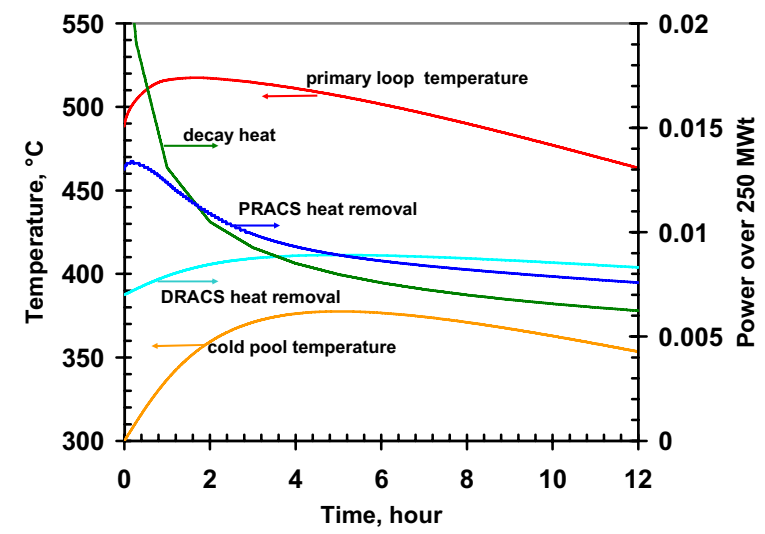

Fig. 3. Lumped-mass transient temperature response of the 250 MWt hybrid SFR to LOFC.

Fig. 4 shows preliminary RELAP5-3D analysis results for LOFC transients, including peak cladding temperature, hot pool temperature and reactor inlet temperature. In this case, the buffer pool temperature is set at the same temperature as the reactor inlet. The primary pump coastdown time used in this analysis is only about half of time for the ABTR design. The hot pool temperature increases to the highest value by about $75^{\circ} \mathrm{C}$ around two hours, which is close to the prediction of lumped-mass based calculation. The peak cladding temperature rises to $660^{\circ} \mathrm{C}$ slightly after the end of pump coast down. The peak clad temperature increase is very mild and within the design limit, which can be compared with the maximum $750^{\circ} \mathrm{C}$ peak clad temperature predicted for the ABTR design $^{19}$. The second peak of the peak cladding temperature arrives around half an hour later at much lower value. Reference 18 presents more detailed information on the RELAP5-3D simulation. 
These results show that the passive heat removal systems are very efficient at transferring decay heat from fast reactor fuels to the hot primary coolant, the cold pool, and the environment. These results also show that additional margin exists and suggest that it may be possible to further increase the normal reactor inlet and outlet temperatures and boost reactor thermal efficiency while maintaining the peak cladding and hot pool temperatures during LOFC transients within safety requirements. Future validation work for the RELAP5-3D SFR simulation and benchmark comparisons with other SFR analysis codes will give uncertainty ranges for these simulation results.

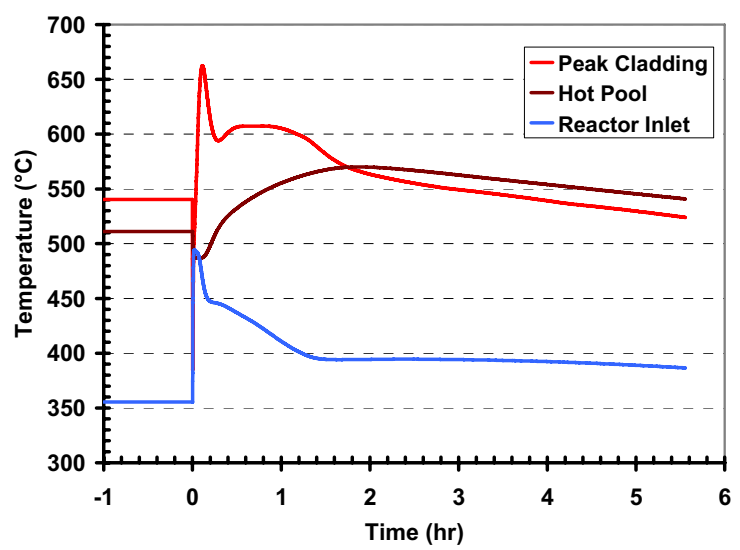

Fig. 4. Temperature variations during LOFC by RELAP5-3D.

\section{MULTIPLE REHEAT HELIUM BRAYTON CYCLES}

\section{III.A. Advanced Helium Brayton Cycles}

Both economics and safety for SFRs are affected by the selection of the power conversion system. Traditionally, the steam Rankine cycle has been the only available power conversion choice for SFRs. The potential for energetic water-sodium reactions is a long-standing issue that at least partially delays the near-term commercialization of SFR technology and is one of the major contributors for the relatively high overall cost of the SFR. For example, to prevent and mitigate sodium water reactions in steam generators, expensive double walled tubing and complex safety systems are used ${ }^{15}$. Concern about sodium reactions remains, as sodium leakage incidents have occurred in a majority of the larger prototype SFRs. Therefore, advanced SFR designs must address the water-sodium reaction safety concern and improve economics. Advanced closed gas Brayton cycles with compatible fluids such as the inert gas helium provide an opportunity to address this issue.

Helium Brayton cycles are chosen for the power conversion cycle in Gen-IV High Temperature Gas Cooled
Reactor (HTGR) designs such as the General Atomics Gas Turbine - Modular Helium Reactor (GT-MHR) ${ }^{20}$ and Pebble Bed Modular Reactor $(\mathrm{PBMR})^{21}$. These power conversion systems have undergone detailed engineering design and further R\&D efforts, particularly for the PBMR, will solve development issues related to closed gas cycles. Net thermal efficiency in the range of $42 \%$ to $46 \%$ is predicted with turbine inlet temperature from $850^{\circ} \mathrm{C}$ to $900^{\circ} \mathrm{C}$. However, current SFRs can only achieve reactor outlet temperatures between $500^{\circ} \mathrm{C}$ to $550^{\circ} \mathrm{C}$ due to the peak metallic clad temperature limit. Even considering longer term advances in materials, the possible reactor outlet temperature may only increase to $650^{\circ} \mathrm{C}$ due to the sodium boiling temperature of $881.4^{\circ} \mathrm{C}$ at $1 \mathrm{~atm}$. Under this range of reactor outlet temperatures, the conventional closed helium Brayton cycle has significantly lower efficiency than steam cycles. One solution to improve the Brayton cycle efficiency can be through multiple reheat and intercooling.

Reheat has been widely used in modern steam cycles. For gas-cooled reactors reheat has not proven to be practical due to the high pressure loss associated with pumping helium to and from the reactor core. Because molten coolants such as sodium can transport heat with low pumping power to compact heat exchangers, reheat becomes an attractive option with molten coolants. By utilizing reheat, these multiple reheat molten coolant gas cycles (MCGC) have the potential for substantially higher thermal efficiency than current gas cooled reactors, if used with comparable turbine inlet temperatures ${ }^{22}$. A MCGC power conversion system (PCS) using heat from AHTR can achieve net thermal efficiency at $56 \%$ for the turbine inlet temperatures of $900^{\circ} \mathrm{C}^{23}$. Very high PCS power density can be achieved in these designs, which could imply large material savings and low construction costs, and reduce the specific PCS cost to about half that of the current GT-MHR PCS design. MCGC designs were also applied for fusion power plant conceptual design. ${ }^{24}$

A recent study extended the multiple reheat helium Brayton cycle design to SFR ${ }^{11}$. Fig. 5 shows a temperatureentropy (T-s) diagram for an optimized MCGC reference design for a SFR with 3 expansion stages and 6 compression stages, which shows the basic idea of a MCGC cycle. With multiple reheat stages, the average heat absorbing temperature is close to the highest heat source temperature; and with multiple cooling stages, the average heat rejection temperature is close to the heat sink temperature. The heat transfer in the recuperator is internal, and does not affect the average heat input and heat rejection temperatures. The cycle efficiency for this optimized reference design shown in this figure is $44 \%$ at a turbine inlet temperature of $550^{\circ} \mathrm{C}\left(\mathrm{T}_{\mathrm{a}}\right.$ in Figure 1). The cycle efficiency considers turbomachinary losses, pressure 
losses, and recuperator effectiveness but does not include other losses such as heat loss, generator and gear box (if used) losses, pumping power for cooling systems, and other plant house load. Note that the heating temperature range (from $T_{c}$ to $T_{a}$ and from $T_{b}$ to $T_{a}$ in Fig. 5) matches the SFR reactor inlet and outlet temperature range very well.

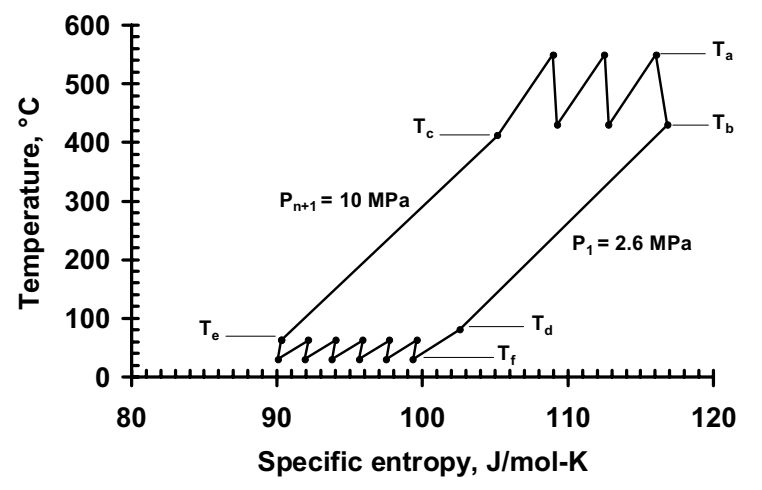

Fig. 5. Temperature-entropy diagram for the MCGC lowtemperature reference case; $\mathrm{T}_{\mathrm{a}}$ is the turbine inlet temperature, $T_{b}$ the turbine outlet temperature, $T_{c}$ the recuperator high-pressure side outlet temperature, $T_{d}$ the recuperator low-pressure side outlet temperature, $\mathrm{T}_{\mathrm{e}}$ the compressor outlet temperature, and $T_{f}$ the compressor inlet temperature.

\section{III.B. Power Conversion Unit (PCU) Arrangement}

There are several major PCU design choices to make when considering multiple-reheat Brayton power conversion systems such as: horizontal shaft versus vertical shaft, single shaft versus multiple shafts, and integrated versus distributed equipment configurations. Previous studies ${ }^{23,25}$ have generated several options for PCU arrangements. Two designs are summarized here.

Fig. 6 shows schematics of an integrated vertical multiple-shaft system design which can be derived from the GT-MHR PCU design. With relatively small engineering modifications, multiple GT-MHR PCUs can be connected together to create a multiple-reheat cycle power conversion system. Approximately $10 \%$ of the cold flow is bypassed upward to flow through an annulus around the hot-leg duct, so the hot leg pressure boundary is maintained at the same temperature as the cold-leg boundary to minimize thermal stresses. The resulting power conversion system is very compact, and results in what is likely the minimum helium duct volume possible for a multiple-reheat system. To do this, compact plate type sodium-to-helium heat exchangers are inserted in the annular space around the turbines, which is currently occupied by the upper set of recuperator heat exchangers in the GT-MHR design; and the recuperator is moved to a separate pressure vessel. Locating heaters in annular arrangement around the turbines gives very short hot-gas flow paths.

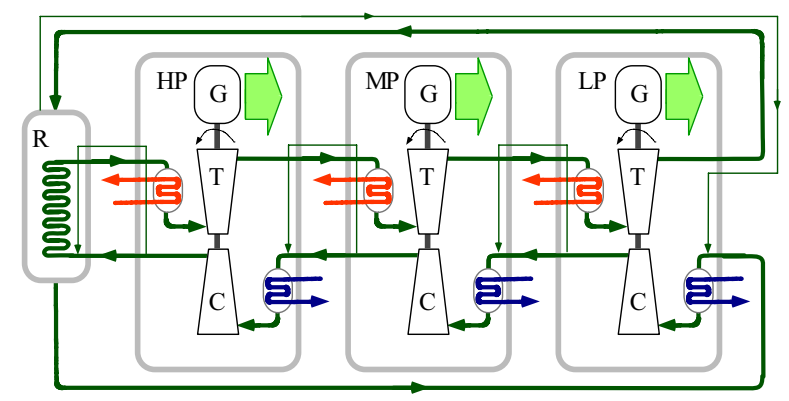

Fig. 6. Schematic flow diagram for the three-expansion-stage multiple-reheat cycle, using three PCU modules (HP, MP, and LP) each containing a generator (G), turbine (T), compressor (C), and heater and cooler heat exchangers, with a recuperator $(\mathrm{R})$ located in a fourth vessel.

Fig. 7 shows the schematic flow diagram for the distributed three horizontal-shaft multiple-reheat cycle, using three PCU modules (HP, MP, and LP) each containing a generator, a turbine, two compressors, and heater and cooler heat exchangers, and a recuperator located in a separate vessel. All the hot ducts are concentric ducts and cooled by cold helium which flows in the annulus outside hot inner ducts. In all these designs, the pressure boundary operates at, or slightly above, the compressor outlet temperature. This is a very low temperature, and thus the pressure boundary can be made from inexpensive materials. There is also a pressure difference between the cold helium next to the pressure boundary and the hot helium in the hot ducts inside, so the hot ducts and turbine casings must operate with some pressure difference and stress too. However, insulation in these systems can allow the hot components to operate closer to the cold temperature than the hot temperature. The steady-state pressure difference between the hot and cold fluids is minimized.

At this stage, it is difficult to select one PCU configuration as the best potential system. But if one considers this choice in the context of broader R\&D efforts on Brayton cycle for nuclear power conversion, one will have clearer pictures for future directions. Fig. 8 compares four different power conversion systems. The top two, the PCUs for GT-MHR and PBMR, are middle power level Brayton cycles without reheat. The bottom two, an integral vertical shaft configuration and another horizontal distributed shaft configuration for SFRs, are large power level Brayton cycles with multiple-reheat. In Fig. 8, the systems on the left are vertical shaft and integrated 
systems; and the systems on the right are horizontal shaft and distributed systems. The success of the GT-MHR, and/or PBMR, program will solve most technology challenges for the development of multiple-reheat systems and will establish the technology base to choose directions for larger multi-reheat systems.

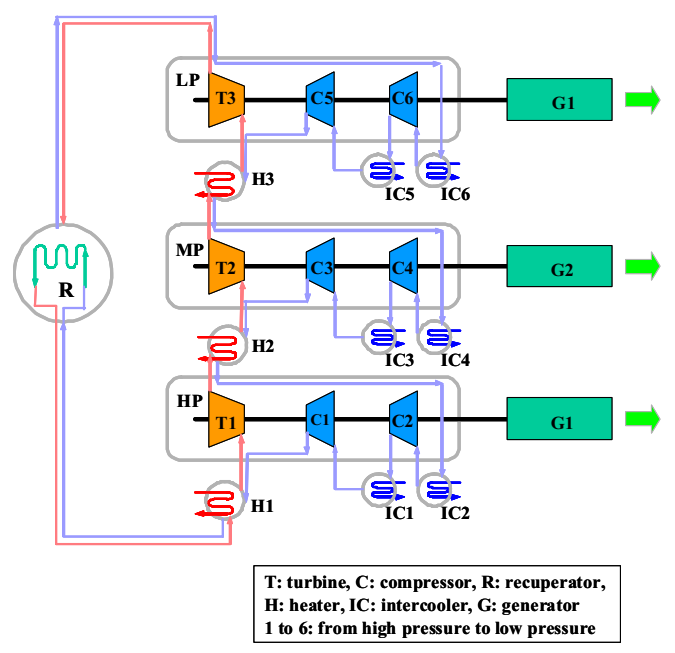

Fig. 7. Schematic flow diagram for the distributed three horizontal-shaft multiple-reheat cycle, using three PCU modules.

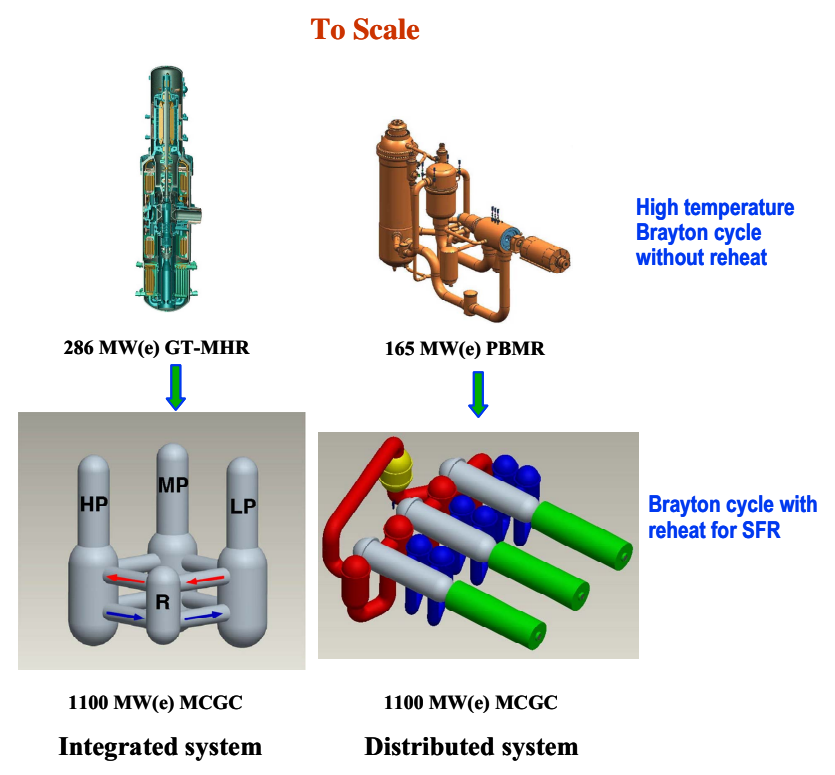

Fig. 8. Scaled comparison of four Brayton power conversion systems.

III.C. Comparison with Other Power Cycles
Due to the safety concerns over steam cycles, alternative power cycles are being explored in new SFR designs. SCO2 recompression Brayton cycles $^{26,27}$ are recently proposed as power conversion systems by several advanced sodium fast reactor designs, such as US ABTR design ${ }^{1}$. This section compares several power cycles, mainly between MCGC and SCO2 Brayton cycles for SFRs. Fig. 9 compares the cycle efficiencies for five types of cycles in the interested turbine inlet temperature range for SFRs. The efficiency data for SCO2 cycles, supercritical and superheated steam cycles are directly taken from references 26 and 27. SCO2 cycle assumes $32^{\circ} \mathrm{C}$ compressor inlet temperature. All other cycles assume $30^{\circ} \mathrm{C}$ compressor inlet temperature except for the MCGC case with $20^{\circ} \mathrm{C}$ compressor inlet temperature. Comparable parameters used in $\mathrm{SCO} 2$ cycles are assumed in the helium Brayton cycle calculations: i.e., the turbine adiabatic efficiency at $93 \%$, the compressor adiabatic efficiency at $88 \%$, and the recuperator effectiveness at $95 \%$. For the MCGC cycles, the total relative pressure loss (the system pressure is $10 \mathrm{MPa}$ ) is $6 \%$. The total pressure loss value was verified by summing all the pressure losses from each component, such as turbines, compressors, heat exchangers, and ducts. The analysis ${ }^{11}$ shows that MCGC with three stages of expansion and six stages of compression (3T6C) tends to be the best balance between thermal efficiency and equipment cost.

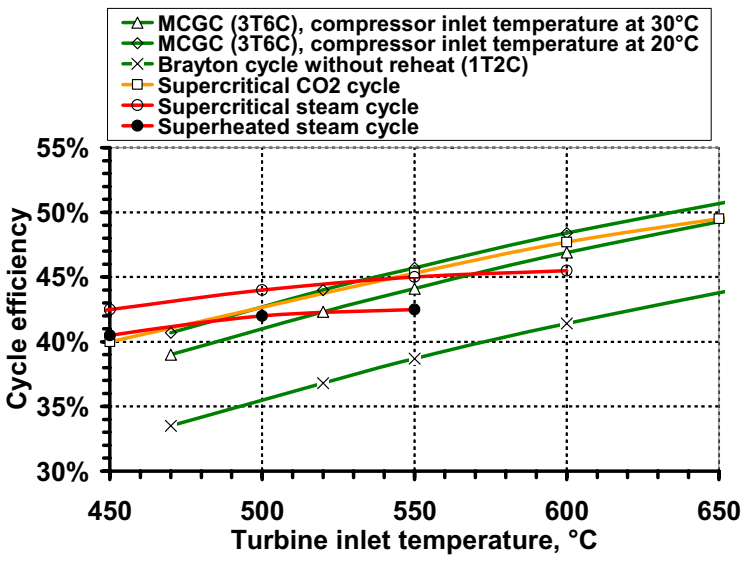

Fig. 9. Comparison of cycle efficiencies of MCGC (3T6C means 3 stages of expansion and 6 stages of compression), helium Brayton cycle without reheat, $\mathrm{SCO} 2$ cycles, superheated and supercritical steam cycles.

Superheated steam cycles are widely used for SFR designs such as $\mathrm{ALMR}^{3}$ (net efficiency at $36 \%$, steam conditions: $15.16 \mathrm{MPa} / 430^{\circ} \mathrm{C}$ ), $\mathrm{EFR}^{2}$ (net efficiency at $40 \%$, steam conditions: $18.5 \mathrm{MPa} / 490^{\circ} \mathrm{C}$ ), $\mathrm{JSFR}^{7}$ (thermal efficiency at $42.5 \%$, steam conditions: $19.2 \mathrm{MPa} / 497^{\circ} \mathrm{C}$ ). Supercritical steam cycles offer about $5 \%$ more efficiency than superheated steam cycles, but require steam pressure above $22.1 \mathrm{MPa}$. Although steam cycles have slightly 
higher thermal efficiency than advanced Brayton cycles such as $\mathrm{SCO} 2$ and MCGC for the turbine inlet temperatures up to $480^{\circ} \mathrm{C}$ for superheated steam cycles and up to $540^{\circ} \mathrm{C}$ for supercritical steam cycles, the compatibility issue related to sodium-water reaction makes water a far less desirable power cycle fluid.

The conventional closed helium Brayton cycle with intercooling has much lower efficiency than the $\mathrm{SCO} 2$ cycle in the interested temperature range, which is the reason why the helium Brayton cycle was not further considered as power conversion choices for SFR in the past. However, when one considers using reheat, the cycle efficiencies for MCGC and SCO2 cycles fall into the same range, with the maximum efficiency difference about $1 \%$, which is within the range of engineering design uncertainty. When considering the effects of variation of heat sink temperature and operation at partial load, the thermal efficiency shifts to favor the MCGC cycle as shown in the later discussion. The following sections discuss several most important comparisons.

\section{Compatibility issue}

Power conversion fluid choice is a fundamental issue for SFR plant design. Heat exchangers between intermediate sodium loop and the power conversion loop have higher potential of leakage for several reasons: high pressure difference, high operation temperature, large heat transfer area, and thin walls to reduce heat transfer resistance. For a large power plant, the probability of leakage increases approximately proportionally with thermal power. The situation is similar as with PWR plants, in which steam generator tube breaks do occur and the failed tubes need to be plugged during outages. For leakage in a sodium steam generator, the situation is totally different due to the violent heat-release reaction between sodium and water. The safety systems dealing with sodium-water reactions in a sodium steam generator increase the capital cost. If the $\mathrm{SCO} 2$ cycle is adopted for SFRs, a similar compatibility problem exists between sodium and $\mathrm{CO}_{2}{ }^{28,29}$ as with water. The energetic reaction of $\mathrm{CO}_{2}$ with sodium will generate even higher temperatures than sodium-water reactions and the major products include $\mathrm{CO}$ and the molten salt of $\mathrm{Na}_{2} \mathrm{CO}_{2}$. $\mathrm{CO}$ is a toxic gas, which may threaten the safety of plant workers; $\mathrm{Na}_{2} \mathrm{CO}_{2}$ could block pipes and contaminate the IHX. If a large amount of sodium reacts with $\mathrm{CO}_{2}$, the released energy may threaten the integrity of the primary loop. Due to the severe nature of the $\mathrm{Na}-\mathrm{CO}_{2}$ reaction, it can be anticipated that expensive and complex safety systems will be required, which may significantly increase the capital cost.
Compared to water and $\mathrm{CO}_{2}$, helium leakage into intermediate sodium loop has no safety implication for the plant and has very little effect on plant availability. The MCGC system operates at much lower pressure (10 MPa versus $20 \mathrm{MPa}$ for $\mathrm{SCO} 2$ cycle), which means a much smaller pressure difference across heaters, and helium is inert gas and does not corrode structure materials while both water and $\mathrm{CO}_{2}$ are corrosive at high temperature. Therefore, the possibility of heat exchanger wall breaks should be much lower for helium. If any wall break does occur, the break area will not increase due to heating from $\mathrm{Na}-\mathrm{H}_{2} \mathrm{O}$ or $\mathrm{Na}-\mathrm{CO}_{2}$ reactions. The leaked helium can be recovered in the expansion tank of the intermediate heat transfer loop. The plant can continue to operate unless the break area becomes too large.

\section{Heat sink temperature}

$\mathrm{SCO} 2$ Brayton cycles take advantage of the unique feature that the compression work is minimized around the $\mathrm{CO}_{2}$ critical point $\left(32^{\circ} \mathrm{C}, 7 \mathrm{MPa}\right)$. This brings high efficiency of $\mathrm{SCO} 2$ cycle. However, this also brings a substantial disadvantage of $\mathrm{SCO} 2$ cycle. Because the $\mathrm{SCO} 2$ cycle must be optimized around the critical point for compressor design, any deviation from this point will strongly affect the efficiency. When the compressor inlet temperature increases from $32^{\circ} \mathrm{C}$ to $50^{\circ} \mathrm{C}$, the cycle efficiency will decrease at a rate about $0.28 \%$ per degree $\mathrm{C}$; when the compressor inlet temperature decreases, the cycle efficiency keeps same or slightly decreases ${ }^{26}$. The MCGC cycle efficiency decreases at a much slower rate, between $0.15 \%$ to $0.18 \%$ per degree $\mathrm{C}$, depending on turbine inlet temperatures ${ }^{11}$. The compressor inlet temperature is directly related to the heat sink temperature, which varies on plant location and varies in time with weather. For example, if dry cooling is used, the heat sink temperature is the atmosphere temperature.

Plentiful plant sites are available to provide effective cooling so that the average compressor inlet temperature can be maintained below $30^{\circ} \mathrm{C}$. These locations include northern inland areas with low annual average temperature or coastal areas with excellent low temperature seawater heat sink. Fig. 9 shows two curves for MCGC cycles with the compressor inlet temperatures at $20^{\circ} \mathrm{C}$ and $30^{\circ} \mathrm{C}$, respectively. In cold areas, helium cycles can increase efficiency rapidly while $\mathrm{SCO} 2$ cycles have difficulty to benefit; in hot areas, $\mathrm{SCO} 2$ cycles will have greater efficiency decrease due to their high sensitivity to compressor inlet temperature. $\mathrm{SCO} 2$ cycles can achieve their optimal efficiency only when the cooling temperature varies inside a narrow range.

When a plant has been built, the heat sink temperature variation affects the thermal efficiency. MCGC can enjoy 
the cold winter weather to increase efficiency and is subjected to weaker penalty on efficiency in the summer. $\mathrm{SCO} 2$ cycle can only keep the optimized efficiency during colder weather and its efficiency decreases more in hotter weather because it is optimized around the $32^{\circ} \mathrm{C}$ critical point. Analysis shows ${ }^{11}$ that the monthly averaged thermal efficiencies can change between $5 \%$ to $10 \%$ from winter to summer if dry cooling is used. Extremely hot weather usually means electricity demand peaking, when $\mathrm{SCO} 2$ cycles depart from their optimal design conditions the most.

\section{Optimization of PCS and overall plant cost}

The PCS cost accounts for 30 to $40 \%$ of the total equipment cost in $\mathrm{LWRs}^{30}$. However, this ratio is lower for current SFR plant designs with steam cycles. The steam turbine plant equipment for SFR only accounts for about $20 \%$ of the total plant $\operatorname{cost}^{27}$. Due to higher power density and elimination of large and bulky equipment items like condensers which operate under sub-atmosphere pressure in steam cycles, the Brayton cycle PCS tends to be much smaller and more compact than steam cycles ${ }^{31}$. Therefore, the cost ratio of Brayton cycle PCS to the total SFR plant cost can be expected to drop further to the range of $10 \%$ to $20 \%$. SCO 2 cycles have higher power density therefore potential lower cost than MCGC cycles. However, due to the small portion of PCS cost to the total SFR plant cost, the factor becomes much less important when selecting cycle types. Additional cost from $\mathrm{Na}-\mathrm{CO}_{2}$ reaction mitigation systems may significantly increase overall cost for a SCO2-SFR plant.

\section{Summary}

Helium Brayton cycles with multiple reheat and intercooling for SFRs with reactor outlet temperature in the range of $510^{\circ} \mathrm{C}$ to $650^{\circ} \mathrm{C}$ can achieve thermal efficiencies between $39 \%$ to $47 \%$, which are comparable with SCO2 cycles. The discussion above clearly shows that the multiple reheat helium cycle could provide major opportunities to improve SFR reliability, safety and economics. The major advantage comes from the use of inert helium as the power conversion working fluid, which eliminates the requirement for safety systems to prevent and mitigate the sodium-water and sodium- $\mathrm{CO}_{2}$ reactions.

\section{HIGH FIDELITY THERMAL HYDRAULICS COMPUTATION}

The SFR economics can also be improved by reducing safety analysis uncertainty and the associated requirements for over-conservatism in design through high fidelity, multi-scale, multi-physics thermal hydraulic analysis methods. From Fig. 9, the MCGC thermal efficiency can be increased in a faster rate than steam cycles by increasing the reactor outlet temperature. However, the peak clad temperature in steady state and safety margins during accidents limit the maximum reactor outlet temperature which can be used with the current fuel design. By reducing analysis uncertainties with high fidelity methods, the steady state reactor outlet temperature could be increased even with current available fuel design and structural materials. Certainly the steady state design also needs to consider transient situations. The optimized hybrid loop-pool SFR design could potentially provide better passive cooling ability so that the reactor safety is not degraded. This section reviews ongoing works about high fidelity multi-physics coupled simulations, uncertainty qualification, and higher dimensional methods.

\section{IV.A High Fidelity Coupled Thermal Hydraulics and Neutronics for Reactor Safety Simulations}

Advances in numerical methods during the past two decades make it possible to perform simulations with second order accuracy in both time and space. New methods also enable implicitly coupled solution algorithms between coolant thermal hydraulics, solid conduction, and neutronics, which can greatly reduce numerical errors in simulations for very fast or very long transients ${ }^{32,33}$.

The traditional reactor system analysis approach is to separately develop a neutronics code (i.e. PARCS, NESTLE) and a thermal hydraulics code (i.e. TRACE, RELAP5) and then to loosely couple them together. Whenever nonlinearly coupled physics are linearized or operator split there are new first order truncation errors that are introduced into the simulation. These truncation errors affect the physics of the simulation. In the operator split approach of coupled physics, one relies heavily on engineering judgment and experimental data to assess the importance of the truncation errors. Often solutions can be "tuned" to match experimental data by accounting for the numerical errors through modifying physical models ${ }^{34}$. For new reactor designs, that do not have a large experimental database supporting them, accuracy of the simulation is critical. The simulation tool will be required to predict the behavior of the reactor when experimental data are limited, and to predict local parameters that cannot reasonably be measured with instruments in experiments. Here one needs accurate simulations with quantified uncertainties that can be used to help in the decision making process.

To accurately quantify the uncertainty of a physical model one needs to make sure that the truncation errors do not play a significant role in the simulation. To accomplish this one needs to make the physics simulation independent of time step and spatial grid resolution. This can be 
achieved with a coupled neutronics thermal hydraulics simulation that is second order accurate in space and time.

One of the efficient numerical methods to solve coupled non-linear equations is physics-based preconditioned Jacobian-free Newton-Krylov method (JFNK) ${ }^{35,36}$. Newton's method requires multiple iterations to converge the nonlinearities in each time step. Each of these nonlinear iterations requires the solution of a matrix. The matrix solution method employed is a Krylov method. The Krylov method does not require the Jacobian matrix; it only requires the product of the Jacobian matrix and a vector. This Jacobian matrix vector product can be approximated by a finite difference formula. This finite difference formula is called the Jacobian-free approximation. The solution to the linear system can therefore be computed without ever having to form the Jacobian matrix. The efficiency of this method depends on the convergence rate of the Krylov iteration. To improve the convergence of the Krylov method one can employ a physics-based preconditioner. The idea behind a physicsbased preconditioner is to use an old linearized and operator split solution method to approximate the answer. Here one combines the efficiency of the linearized and operator split solution method with the accuracy of a Newton's method.

A pilot system analysis code (called System Analysis for Reactor Applications with High Fidelity, SARAH) is being developed at INL to implement the ideas above. ${ }^{12}$ The goal of this work is to improve the accuracy of nuclear reactor safety transient analyses by developing a single code that uses a single nonlinear solution method to solve the nonlinearly coupled equations of thermal hydraulics and neutronics simultaneous. By having a tightly coupled simulation code that has no linearizations or operator splitting and is second order accurate in space and time, one can address the importance of the truncation errors on the safety transient analyses.

Fig. 10 shows the importance of the 1st order truncation errors as a simplified example. In this simulation a simplified primary system model of the ABTR is modeled (see the left figure in Fig. 1). This transient is isothermal with no momentum loss. The hot pool level is initialized higher than the cold pool level. This potential energy is converted to kinetic energy as the sodium flows from the hot pool to the cold pool raising the height of the cold pool and being converted back into potential energy. Since there are no momentum loss terms and no energy sources or sinks, the analytical solution is that total energy is conserved. The heights of the hot pool and cold pool keep oscillating periodically without any damping effect.
The red data set is the 2 nd order in time method run with a time step of $1 / 2$ seconds and this does a good job at preserving total energy since there are no 1 st order truncation errors modifying the solution. The green lines are the 1st order in time results for time steps of $1 / 2$ and $1 / 16$ seconds. The results clearly show that the 1 st order in time method damps the magnitude of the height oscillation of the hot pool and the cold pool. As the time step is made smaller the 1st order truncation error becomes smaller and the 1 st order method approaches the correct solution. These initial results indicate that the first order truncation errors can be an important part of a simulation.

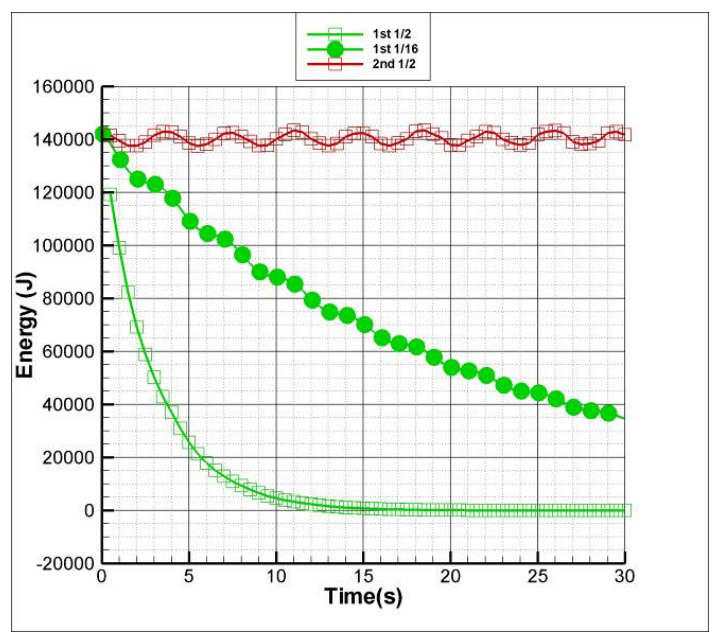

Fig. 10 Effect of 1 st order in time truncation error.

\section{IV.B Uncertainty Quantification}

Progress in verification and validation (V\&V) in CFD fields ${ }^{37}$ and from broader software engineering fields makes it possible to obtain high confidence in new high fidelity software. For example, order-of-accuracy verification in a 3-D CFD code has been performed through method of manufactured solution $(\mathrm{MMS})^{38}$ to verify second order accuracy and identify the existence of any first order errors. Advances in sensitivity analysis techniques ${ }^{39,40,41}$ can be utilized to quantify uncertainties, which is meaningful only after rigorous $V \& V$ has guaranteed that the numerical errors are small.

The traditional approach ${ }^{42}$ to uncertainty quantification is based on a "black box" approach. The simulation tool is treated as an unknown signal generator, a distribution of inputs is sent in and the distribution of the output is measured and correlated back to the original input distribution. This approach is usually performed with coarse resolution models in space and time because of the larger number of simulation runs required to resolve the distribution of inputs. Even with coarse resolution models, 
the total computation cost of this method is still very high due to the requirement of many runs.

Contrary to the "black box" method, a more efficient sensitivity approach can take advantage of intimate knowledge of the simulation code. In this approach equations for the propagation of uncertainty are constructed and the uncertainty / sensitivity are solved for as variables in the simulation. This can generate similar sensitivity information as the above "black box" approach with a single run. Because only one single run is required, this run can be done with a high accuracy in space and time ensuring that the uncertainty of the physical model is being measured and not simply the numerical error caused by the coarse discretization. Because of the increased efficiency and accuracy of this method, the uncertainty of many physical models can be measured and ordered according to uncertainty scales. Given this quantitative measure of uncertainty scales, one can prioritize the effort of model improvement according to where the model improvement will result in the largest reduction of uncertainty.

There are two sensitivity analysis methods available: forward sensitivity method and adjoint sensitivity method ${ }^{41}$. In the forward sensitivity method, the model is differentiated with respect to each parameter to yield an additional system of the same size as the original one, the result of which is the solution sensitivity. The gradient of any output variable depending on the solution can then be directly obtained from these sensitivities by applying the chain rule of differentiation. The forward sensitivity method is mostly suitable when one needs the gradients of many outputs with respect to relatively few parameters. In the adjoint method, the solution sensitivities need not be computed explicitly. Instead, for each output variable of interest, one forms and solves an additional system, adjoint to the original one, the solution of which can then be used to evaluate the gradient of the output variable with respect to any set of model parameters. The adjoint sensitivity method is more practical than the forward approach when the number of parameters is large and when one needs the gradients of only few output variables.

\section{IV.C Higher Dimensional Computation}

Other progresses such as higher dimensional methods than the zero or one dimensional methods used by conventional system codes could also reduce uncertainties, i.e., CFD simulations for components with strong 3-D effects and 1-D transient models for large volume thermal stratification.

3-D whole core analysis with better inter-subassembly mixing and heat transfer models and 3-D CFD simulation for a complete sub-assembly could reduce hot channel or hot spot factors so that more accurate steady state peak inner wall cladding temperature could be predicted. The maximum inner wall cladding temperature is one of major design constrains for SFRs, which directly affects how high the reactor outlet temperature can be in steady state.

At steady state, the nominal temperature drop across the clad is about 30 to $50^{\circ} \mathrm{C}$; the nominal film temperature drop is about 10 to $20^{\circ} \mathrm{C}$; the nominal coolant temperature increase from reactor inlet to the hot spots is around 100 to $150^{\circ} \mathrm{C}$ (according to Todreas and Kazimi ${ }^{43}$, and RELAP53D simulation results). Non-statistical combined hot channel factors at $3 \sigma$ confidence level for the coolant temperature and the film temperature are about 1.2 and 1.3, respectively ${ }^{43}$. Among them, the combined hot channel factor for the coolant temperature due to sub-assembly flow and heat transfer calculation uncertainties is about 1.15 , which is equivalent to approximately a $25^{\circ} \mathrm{C}$ temperature difference; the combined hot channel factor for the film temperature due to sub-assembly flow and heat transfer calculation uncertainties is about 1.25 , which is equivalent to a $5^{\circ} \mathrm{C}$ temperature difference. The combined hot spot effect due to subassembly calculation uncertainty is equivalent to an increase of about $30^{\circ} \mathrm{C}$ in the peak clad temperature, which could be reduced with more accurate simulation. Therefore, a higher reactor outlet temperature than current design used can be used to increase thermal efficiency. For example, when the reactor outlet temperature increases from $540^{\circ} \mathrm{C}$ to $570^{\circ} \mathrm{C}$, the MCGC thermal efficiency can increase by about $4 \%$.

Reynolds Average Navier-Stokes (RANS) CFD simulations are now widely used in engineering calculations. Although RANS simulations depend on experiments or more complicated turbulence simulations to provide closure model data in order to obtain reliable results, they can still provide valuable insights for complex 3-D flow and temperature distribution within a SFR subassembly ${ }^{44}$. Direct Numerical Simulation of Turbulence (DNS) has been attempted to simulate flow around a single rod within a tight lattice fuel pin subassembly by assuming isothermal and incompressible conditions ${ }^{45}$. Due to computing power limits, it will remain impossible to perform whole sub-assembly DNS simulation in the near future. However, the insights gained from future single rod non-isothermal DNS simulations can provide better sub-grid models for coarse turbulence modeling such as RANS or large eddy simulations. CFD simulations already show that there exists better thermal mixing within a subassembly than traditional methods assume. Ongoing efforts on SFR subassembly and whole core level CFD simulation will eventually help to reduce hot spot factors so that reactor outlet temperature could be increased. 
Another important phenomenon in pool type SFR design and analysis is thermal stratification in pools. It is important to accurately predict the temperature distributions both for design optimization and accident analysis. Current major system analysis codes used for SFRs only provide lumped-volume based models which can only give very approximate results and can only handle simple cases with one mixing source. While 2-D or 3-D CFD methods can be used to analyze simple configurations, these methods require very fine grid resolution to resolve thin substructures such as jets and wall boundaries, yet such fine grid resolution is difficult or impossible to provide for studying the reactor response to transients due to computational expense, particularly for mixing by free plumes. Therefore, new methods are needed to support design optimization and safety analysis of pool type SFR systems.

Previous scaling has shown that stratified mixing processes in large stably stratified enclosures can be described using one-dimensional differential equations, with the vertical transport by free and wall jets modeled using standard integral techniques ${ }^{46}$. This allows very large reductions in computational effort compared to threedimensional numerical modeling of turbulent mixing in large enclosures. The BMIX++ (Berkeley mechanistic MIXing code in $\mathrm{C}++$ ) code was originally developed at UC Berkeley to implement such ideas ${ }^{47,48}$, 49 . This code solves transient mixing and heat transfer problems in stably stratified enclosures. The code uses a Lagrangian approach to solve 1-D transient governing equations for the ambient fluid and uses analytical or 1-D integral models to compute substructures such as circular/line buoyant jets, wall boundary flows/wall jets, etc. 1-D solid conduction model is also included in the code.

Various problems with different combinations can be solved by the BMIX++ code, such as: multi-component fluid, variable enclosure cross section area in vertical direction, multi-enclosures, and multiple jets, plumes, and sinks in one enclosure. Available substructure models in the current version include curved free circular buoyant jets, vertical line buoyant jets, isothermal wall jets, and boundary wall conductions.

By including liquid salt properties, the BMIX++ code was recently extended to analyze liquid salt pool systems in the AHTR design, which has similar buffer pool configuration to the hybrid SFR design. ${ }^{50}$ Figure 11 shows the temperature profile in the AHTR buffer salt pool, as calculated by the BMIX++ code. Due to the competing effects by one group of upward hot buoyant jets, one group of downward cold buoyant jets, and two groups of mass sinks, there are two thermal fronts: the upper one for the hot jets and the lower one for the cold jets. Thermal stratification in the buffer salt is divided into three regions: the top region above the PHX, the lower region below the DHX, and the middle one between the PHX and DHX. Under LOFC transient conditions, the PHX heating power increases and the driving force for thermal stratification becomes stronger. Therefore, the thermal stratification in the buffer salt becomes larger. In Fig. 11, the thermal front profiles are very sharp. Similar analysis can be performed for SFRs and the BMIX++ code can be coupled with a system analysis code to provide better prediction of thermal stratification in pool systems.

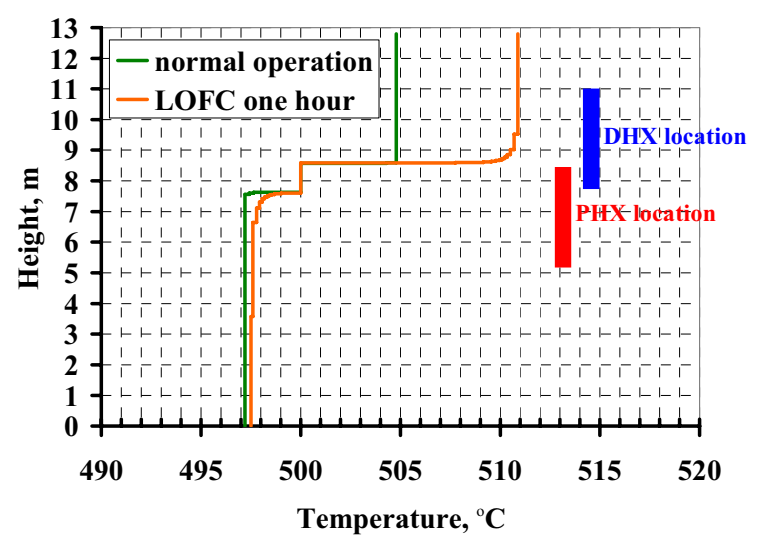

Fig. 11 Temperature profiles in AHTR buffer salt tank for steady state operation, and for LOFC at one hour.

\section{CONCLUSIONS}

Innovations from the perspective of thermal hydraulics provide potential opportunities to improve SFR economics. The new hybrid loop-pool reactor design can potentially help further optimize SFR designs so that safety, reliability, and cost reduction goals can be achieved simultaneously. A multiple reheat and intercooling helium Brayton cycle eliminates the issue of chemical reactions between sodium and water or between sodium and $\mathrm{CO}_{2}$, and has much more compact configuration of power conversion equipment compared to steam cycles. Modern multi-physics thermal analysis methods can reduce analysis uncertainties and associated requirements for over-conservatism in reactor design. It can be expected that benefits on SFR economics can be maximized with the combination of all these innovations together.

\section{ACKNOWLEDGMENTS}

This work was supported through INL Laboratory Directed Research and Development program under DOE Idaho Operations Office Contract DE-AC07-05ID14517. 


\section{REFERENCES}

1. Y.I. Chang, et. al., Advanced Burner Test Reactor Preconceptual Design Report, Argonne National Laboratory, ANL-ABR-1 (ANL-AFCI-173), Sept. 5 (2006).

2. J.C. Lefevre, C.H. Mitchell, G. Hubert, "European Fast Reactor Design," Nuclear Engineering and Design, Vol. 162, pp 133-143 (1996).

3. E.L. Gluekler, "U.S. Advanced Liquid Metal Reactor (ALMR)," Progress in Nuclear Energy, Vol. 31, No. 1, pp. 43-61 (1997).

4. L.N. Salerno, et. al., "PRISM Concept, Modular LMR Reactors," Nuclear Engineering and Design, Vol. 109, pp. 79-86 (1988).

5. IAEA, Status of Liquid Metal Cooled Fast Reactor Technology, IAEA-TECDCC-1083, ISSN 1011-4289, (1999).

6. Y. Shimakawa, et. al., "An Innovative Concept of a Sodium-Cooled Reactor to Pursue High Economic Competitiveness," Nuclear Technology, Vol. 140, No. 1, pp. 1-17 (2002).

7. M. Konomura and M. Ichimiya, "Design Challenges for Sodium Cooled Fast Reactors," Journal of Nuclear Materials, Vol. 371, pp. 250-269 (2007).

8. N.E. Todreas, "Thermal Hydraulic Challenges in Fast Reactor Design," Proceedings of the $12^{\text {th }}$ International Topic Meeting on Nuclear Thermal Hydraulics (NURETH-12), American Nuclear Society, Pittsburgh, Pennsylvania, USA (2007).

9. D.C. Crawford, et. al., "Fuels for Sodium-Cooled Fast Reactors: US Perspective," Journal of Nuclear Materials, Vol. 371, pp. 202-231 (2007).

10. H. Zhao, and H. Zhang, "An Innovative Hybrid LoopPool Design for Sodium Cooled Fast Reactor," Transactions of the American Nuclear Society, Vol. 97 (2007).

11. H. Zhao, and P.F. Peterson, "Multiple Reheat Helium Brayton Cycles for Sodium Cooled Fast Reactors," Nuclear Engineering and Design, in press.

12. H. Zhang, V.A. Mousseau, and H. Zhao, "Development of High Fidelity System Analysis Code for GEN IV Reactors," Proceedings of 2008 International Congress on Advances in Nuclear Power
Plants (ICAPP'08), American Nuclear Society, Anaheim, CA, USA (2008).

13. P.F. Peterson, and H. Zhao, "A Flexible Base-Line Design for the Advanced High-Temperature Reactor Utilizing Metallic Reactor Internals (AHTR-MI)," Proceedings of, 2006 International Congress on Advances in Nuclear Power Plants (ICAPP '06), American Nuclear Society, Reno, NV, USA (2006).

14. T. Aoyama, et al., "Core Performance Tests for the JOYO MK-III Upgrade," Nuclear Engineering and Design, Vol. 237, pp. 353-368 (2007).

15. M. Matsuura, et al., "Design and Modification of Steam Generator Safety System of FBR MONJU," Nuclear Engineering and Design, Vol. 237, n 12-13, pp. 1419-1428 (2007).

16. H. Niwa, et al., "Current Status and Perspective of Advanced Loop Type Fast Reactor In Fast Reactor Cycle Technology Development Project," Proceedings of GLOBAL 2007, Advanced Nuclear Fuel Cycles and Systems, American Nuclear Society, pp. 62-70, Boise, Idaho, USA (2007).

17. Leonard J. Koch, Experimental Breeder Reactor-II (EBR-II), An Integrated Experimental Fast Reactor Nuclear Power Station, ANL.

18. H. Zhang, H. Zhao, C.B. Davis, and M. Memmott, "RELAP5 Analysis of Hybrid Loop-Pool Design for Sodium Cooled Fast Reactors," Proceedings of 2008 International Congress on Advances in Nuclear Power Plants (ICAPP'08), American Nuclear Society, Anaheim, CA, USA (2008).

19. F.E. Dunn, T.H. Fanning, and J.E. Cahalan, Preliminary safety evaluation of the advanced burner test reactor, Argonne National Laboratory, ANLAFCI-172, Sept. 15 (2006).

20. M.P. Labar, “The Gas Turbine-Modular Helium Reactor: A Promising Option for Near Term Deployment," Proceedings of 2002 International Congress on Advanced Nuclear Power Plants (ICAPP '02), American Nuclear Society, Hollywood, Florida, USA (2002).

21. R.A. Matzie, "Pebble Bed Modular Reactor (PBMR) Project Update," Proceedings of 2004 International Congress on Advances in Nuclear Power Plants (ICAPP '04), American Nuclear Society, Pittsburgh, Pennsylvania, USA (2004). 
22. P.F. Peterson, "Multiple-Reheat Brayton Cycles for Nuclear Power Conversion with Molten Coolants," Nuclear Technology, Vol. 144, pp. 279-288 (2003).

23. H. Zhao, and P.F. Peterson, "Optimization of Advanced High-Temperature Brayton Cycles with Multiple Reheat Stages," Nuclear Technology, Vol. 158, pp. 145-157 (2007).

24. H. Zhao, G. Fukuda, R. Abbott, and, P.F. Peterson, "Optimized Helium-Brayton Power Conversion for Fusion Energy Systems," Fusion Science and Technology, Vol. 47, pp. 460-466 (2005).

25. H. Zhao, and P.F. Peterson, "Low-Temperature Multiple-Reheat Closed Gas Power Cycles for the AHTR and LSFR," Proceedings of, 2006 International Congress on Advances in Nuclear Power Plants (ICAPP '06), American Nuclear Society, Reno, NV, USA (2006).

26. V. Dostal, P. Hejzlar, and M.J. Driscoll, "High Performance Supercritical Carbon Dioxide Cycle for Next-Generation Nuclear Reactors," Nuclear Technology, Vol. 154, pp. 265-282 (2006).

27. V. Dostal, P. Hejzlar, and M.J. Driscoll, "The Supercritical Carbon Dioxide Power Cycle: Comparison with Other Advanced Power Cycles," Nuclear Technology, Vol. 154, pp. 283-301 (2006).

28. M. Mito, et. al., "Fast Reactor with Indirect Cycle System of Supercritical CO2 Gas Turbine Plant," Proceedings of 2006 International Congress on Advances in Nuclear Power Plants (ICAPP '06), American Nuclear Society, Reno, NV, USA (2006).

29. J.H. Choi, et. al., "Capsule Test for Investigating Sodium-Carbon Dioxide Interaction," Proceedings of 2006 International Congress on Advances in Nuclear Power Plants (ICAPP '06), American Nuclear Society, Reno, NV, USA (2006).

30. R. H. Bryan, and I. T. Dudley, "Estimated Quantities of Materials Contained in a 1000-MW(e) PWR Power Plant," Oak Ridge National Laboratory, ORNL-TM4515 (1974).

31. P.F. Peterson, and H. Zhao, "Material Input for Advanced Brayton Cycle Power Conversion Systems," Transactions of the American Nuclear Society, Vol. 91, pp 420-421 (2004).

32. V.A. Mousseau, "Accurate Solution of the Nonlinear PDE's from Thermal Hydraulics," Nuclear Technology, Vol. 158, pp. 26-35 (2007).
33. M.A. Pope and V.A. Mousseau, "Accuracy and Efficiency of a Coupled Neutronics and Thermal Hydraulics Model," Proceedings of the 12th International Topical Meeting on Nuclear Reactor Thermal Hydraulics (NURETH-12), American Nuclear Society, Pittsburgh, Pennsylvania, USA (2007).

34. N. Zuber, "The Effects of Complexity, of Simplicity and of Scaling in Thermal Hydraulics," Nuclear Engineering and Design, Vol. 204, pp. 1-27 (2001).

35. D.A. Knoll and D.E. Keyes, "Jacobian-free NewtonKrylov Methods: A Survey of Approaches and Applications," Journal of Computational Physics, Vol. 193, pp. 357-397 (2004).

36. V.A. Mousseau, "A fully implicit hybrid solution method for a two-phase thermal-hydraulic model," Journal of Heat Transfer, Vol. 127, pp. 531-539 (2005).

37. W.L. Oberkampf and T.G. Trucano, "Verification and Validation in Computational Fluids Dynamics," Progress in Aerospace Sciences, Vol. 38, pp. 209-272 (2002).

38. R.B. Bond, et. al., "Manufactured Solution for Computational Fluid Dynamics Boundary Condition Verification," AIAA Journal, Vol. 45, No. 9, pp. 22242236 (2007).

39. M.D. Tocci, "Sensitivity Analysis of Large-Scale Time Dependent PDEs," Applied Numerical Mathematics, Vol. 37, pp. 109-125 (2001).

40. S.L. Lee, C.S. Woodward, F. Graziani, "Analyzing Radiation Diffusion Using Time-Dependent Sensitivity-Based Techniques," Journal of Computational Physics, Vol. 192, pp. 211-230 (2003).

41. A.C. Hindmarsh, et. al., "SUNDIALS: Suite of Nonlinear and Differential/Algebraic Equation Solvers," ACM Transactions on Mathematical Software, Vol. 31, No. 3, pp. 363-396 (2005).

42. L.P. Pagani, G.E. Apostolakis, and P. Hejzlar, "The Impact of Uncertainties on the Performance of Passive Systems," Nuclear Technology, Vol. 149, pp. 130-140 (2005).

43. N.E. Todreas and M.S. Kazimi, Nuclear System II Elements of Thermal Hydraulic Design, chapter 8, Hemisphere Publishing Corporation (1990). 
44. R. Gajapathy, et. al., "CFD Investigation of Helical Wire-Wrapped 7-Pin Fuel Bundle and the Challenges in Modeling Full Scale 217 Pin Bundle," Nuclear Engineering and Design, Vol. 237, Issue 24, pp. 23322342 (2007).

45. H. Ninokata and E. Merzari, "Computational Fluid Dynamics and Simulation-Based Design Approach for Tight Lattice Nuclear Fuel Pin Subassemblies," Proceedings of the 12th International Topical Meeting on Nuclear Reactor Thermal Hydraulics (NURETH12), American Nuclear Society, Pittsburgh, Pennsylvania, USA (2007).

46. P.F. Peterson, "Scaling and Analysis of Mixing in Large, Stratified Volumes," International Journal of Heat and Mass Transfer, Vol. 37, Suppl. 1, pp. 97-106 (1994).

47. J. Christensen and P.F. Peterson, "A One-Dimensional Lagrangian Model for Large-Volume Mixing," Nuclear Engineering and Design, Vol. 204, pp. 299320 (2001).

48. H. Zhao, Computation of Mixing in Large Stably Stratified Enclosures, Ph.D. dissertation, University of California, Berkeley (2003).

49. F. Niu, H. Zhao, P.F. Peterson, J. Woodcock, and, R.E. Henry, "Investigation of Mixed Convection in a Large Rectangular Enclosure," Nuclear Engineering and Design, Vol. 237, pp. 1025-1032 (2007).

50. H. Zhao and P.F. Peterson, "One-Dimensional Analysis of Thermal Stratification in AHTR and SFR Coolant Pools," Proceedings of the $12^{\text {th }}$ International Topic Meeting on Nuclear Thermal Hydraulics (NURETH-12), American Nuclear Society, Pittsburgh, Pennsylvania, USA (2007). 